\title{
ESTIMATION OF THE COMPONENTS OF HETEROSIS
}

\author{
J. L. JINKS AND R. MORLEY JONES
}

A.R.C. Unit of Biometrical Genetics, Department of Genetics, University of Birmingham

Received June 17, 1957

\begin{abstract}
$T^{N}$ previous papers (JINKs 1954,1956$)$ the analysis of the parents, $F_{1}$ 's, $F_{2}$ 's and 1 backcross generations of an $8 \times 8$ diallel between inbred varieties of Nicotiana rustica using the method of diallel analysis described by JiNKs and HAYman (1953), and extended by Jinks (1954), Hayman (1954), Dickinson and JiNKs (1956) and Jrnks (1956) have been presented. They showed that nonallelic interactions, as well as additive and dominance effects, play an important role in the inheritance of all the three characters followed, namely, final height, time of flowering and leaf size. This finding was subsequently confirmed for a number of diallel sets of crosses in other species where a wide range of characters were followed (Jinks 1955; ALLARD 1956). What is more important to our present discussion, however, was the finding that the $F_{1}$ generation of crosses showing nonallelic interactions were in general superior in their performance to those of noninteracting crosses. This appears to implicate nonallelic interactions as a major source of heterosis, and we shall now attempt to assess the magnitude of their contribution relative to those of the other components of heterosis.
\end{abstract}

\section{The components of heterosis}

If we define heterosis as the difference between the mean of an $F_{1}$ family and that of its better parent, the expectations can be expressed in terms of the genetic parameters for additivity $(d)$ and dominance $(h)$ (MATHER 1949) and the nonallelic interaction components $(i, j$, and $l$ ) of Hayman and Mather (1955) together with parameters expressing the degree of association or dispersion of the genes in the homozygous parents.

Consider first the case in which all genes have similar effects, and all pairs of genes similar interactions. Let the parents $P_{1}$ and $P_{2}$ differ at $k$ loci, and of these let $P_{2}$ have $k^{\prime}$ of greater effect. Of the $1 / 2 k(k-1)$ pairs of loci, $k^{\prime}\left(k-k^{\prime}\right)$ are dispersed and the remainder associated. The mean parental phenotypes are then

$$
\begin{aligned}
& \overline{\frac{P_{1}}{P_{2}}}= \pm\left(k-k^{\prime}\right) d \mp k^{\prime} d+\left[1 / 2 k(k-1)-k^{\prime}\left(k-k^{\prime}\right)\right] i-k^{\prime}\left(k-k^{\prime}\right) i \\
& \mp 1 / 2\left(k-2 k^{\prime}\right)(k-1) j+1 / 8 k(k-1) l \text {. }
\end{aligned}
$$

If, now, we write $r=1-\frac{2 k^{\prime}}{k}$

this becomes $\frac{\overline{P_{1}}}{P_{2}}= \pm r \Sigma d+\frac{k r^{2}-1}{k-1} \Sigma i \mp r . \Sigma 1 / 2 j+\Sigma 1 / 4 l$. 
$r$ may be taken as a measure of the degree of association for if $r=1, p_{1}$ contains all the genes of greater effect, and if $r=-1, P_{2}$ contains all the genes of greater effect. In either case there is complete association. If on the other hand $r=0$, $k^{\prime}=1 / 2 k$ so that the genes of greater effect are equally shared between $P_{1}$ and $P_{2}$, giving the maximum possible dispersion.

It is convenient to write the equation for the mean parental phenotype more shortly as

$$
\begin{aligned}
& \frac{\overline{P_{1}}}{P_{2}}= \pm[d]+[i] \mp 1 / 2[j]+1 / 4[l] \\
& \quad \text { where }[d]=r \Sigma d,[i]=\frac{k r^{2}-1}{k-1} \Sigma i,[j]=r \Sigma j,[l]=\Sigma l .
\end{aligned}
$$

Writing in addition

$$
[h]=\Sigma h
$$

we have (c.f. Hayman and Mather 1955 Table 3)

$$
\begin{array}{ll}
\overline{B_{1}}=1 / 2( \pm[d]+[h]+1 / 2[i]) & \overline{F_{2}}=1 / 2[h] \\
\overline{F_{1}}=[h]+1 / 4[l] & \overline{F_{3}}=1 / 4([h]+1 / 4[l])
\end{array}
$$

The scaling tests become

$$
\begin{aligned}
& A=\overline{P_{1}}+\overline{F_{1}}-2 \overline{B_{1}}=1 / 2([i]-[j]+[l]) . C=\overline{P_{1}}+\overline{P_{2}}+2 \overline{F_{1}}-4 \overline{F_{2}}=2[i]+[l] \\
& B=\bar{P}_{2}+\bar{F}_{1}-2 \overline{B_{2}}=1 / 2([i]+[j]+[l]) . D=\bar{P}_{1}+\bar{P}_{2}+2 \overline{2 F}_{2}-4 \bar{F}_{3}=2[i]+1 / 4[l]
\end{aligned}
$$

The quantities $[d],[h],[i],[j]$ and $[l]$ may be estimated using the following relations.

$$
\begin{array}{lc}
{[d]=\overline{B_{1}}-\overline{B_{2}}} & {[j]=2 \overline{B_{1}}-\overline{P_{1}}-2 \overline{B_{2}}+\overline{P_{2}}} \\
{[h]=\overline{F_{1}}-4 \overline{F_{2}}-1 / 2 \overline{P_{1}}-1 / 2 \overline{P_{2}}+2 \overline{B_{1}}+2 \overline{B_{2}}} \\
{[i]=2 \overline{B_{1}}+2 \overline{B_{2}}-4 \overline{F_{2}}} & {[l]=\overline{P_{1}}+\overline{P_{2}}+2 \overline{F_{1}}+4 \overline{F_{2}}-4 \overline{B_{1}}-4 \overline{B_{2}}}
\end{array}
$$

The better parent is

$$
\overline{P_{0}}=|[d]-1 / 2[i]|+[i]+1 / 4[l]
$$

where $|[d]-1 / 2[j]|$ means the absolute value, without regard to sign, of $[d]$ $-1 / 2[j]$. Hence measure of heterosis is

$$
\begin{aligned}
& \overline{F_{1}}-\overline{P_{0}}=|[h]-[i]|-|[d]-1 / 2[j]| \\
& =[h]-[i]-[d]+1 / 2[i] \text { if }[h]>[i] \text { and }[d]>1 / 2[i] \\
& =[h]-[i]+[d]-1 / 2[j] \cdots \cdots \cdot \cdots \quad \cdot \quad \cdot \quad[d]<1 / 2[j] \\
& =-[h]+[i]-[d]+1 / 2[i] \cdots[h]<[i] \cdots[d]>1 / 2[i]
\end{aligned}
$$

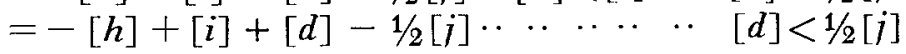


When the condition that different loci shall have equal effects is relaxed, it becomes necessary to take account of the differences in effect when assessing the degree of association. A locus at which two possible allelomorphs had almost identical effects could clearly make only a small contribution to the effective degree of association or dispersion. However, all the above formulae remain valid except those connecting $[d],[i]$ and $[i]$ with the basic model.

This is easily seen if we write $d_{a}{ }^{*}= \pm d_{a}$ and $\left.j_{a}\right|_{b}{ }^{*}= \pm\left. j_{a}\right|_{b}$ according as $P_{1}$ or $P_{2}$ carries the gene of greater average effect at the $A$ locus, and $i_{a b}= \pm i_{a b}$ according as the genes at the loci $A$ and $B$ are associated or dispersed. Then

$$
\begin{aligned}
& \overline{P_{1}}= \pm \Sigma d^{*}+\Sigma i^{*} \mp 1 / 2 \Sigma j^{*}+1 / 4 \Sigma l \\
& \overline{P_{2}}=1 / 2\left(\Sigma d^{*}+\Sigma h\right)+1 / 4 \Sigma i^{*}
\end{aligned}
$$

etc., and the formulae are of the same form as those described above for the special case of equal genic effects, except that we now have

$$
[d]=\Sigma d^{*},[h]=\Sigma h,[i]=\Sigma i^{*},[j]=\Sigma j^{*} \text { and }[l]=\Sigma l \text {. }
$$

An equivalent form is

$$
[d]=r_{d} \Sigma d,[i]=q_{i} \Sigma i,[j]=r_{j} \Sigma j
$$

where now $r_{d}$ and $r_{j}$ are the degree of association reckoned as before, except that each locus $A$ is assigned a score equal to $d_{a}$ or $\left.\Sigma_{b} \dot{j}_{a}\right|_{b}$ respectively, instead of simply 1 , when the number of loci are being counted. Similarly, $q_{i}$ is the proportion of associated pairs minus that of the dispersed pairs, the counting being carried out with scores equal to $i$. Since some of the $i$ 's and $j$ 's may be negative, $q_{i}$ and $r_{j}$ are not restricted to being less than 1 in absolute value.

\section{Classification of nonallelic interactions}

For two interacting genes the relationship between the parameters for nonallelic interactions and the classical interactions have been given by HAYMAN and MATHer (1955). For example, classical complementary genes are given by the relationship $d_{a}=d_{b}=h_{a}=h_{b}=3 / 2 \quad\left(i_{a b}=\left.j_{a}\right|_{b}=\left.j_{b}\right|_{a}=l_{a b}\right)$ and duplicate genes by $d_{a}=d_{b}=h_{a}=h_{b}=-1 / 2 \quad\left(i_{a b}=\left.j_{a}\right|_{b}=\left.j_{b}\right|_{a}=l_{a b}\right)$ while other classical interactions can be derived from these by inequality of $d_{a}$ and $d_{b}$.

The classical interaction can, however, be divided into two groups, those where the over-all effects of the nonallelic interactions reinforce the effects of dominance, e.g. complementary genes, recessive epistasis, etc., and those where the two effects are opposing, e.g., duplicate genes, dominant epistasis, etc. Where we are dealing with the sum or balance of the effects of many genes, inequalities in the contribution of individual genes (such as distinguish complementary genes and recessive epistasis or duplicate genes and dominant epistasis) would be difficult or impossible to detect: the only feature of the classical classification we can profitably utilize, therefore, is the distinction between reinforcing and opposing interactions. These will be referred to as complementary and duplicate type interactions respectively.

In practise the classification must primarily depend on the relative signs of 
[h] and [l] as only these two components of the generation means are independent of the degree of association, which can itself take sign, and hence affect the sign of $[d],[i]$ and [j] independently of the sign of $\Sigma d, \Sigma j$ and $\Sigma i$.

\section{OBSERVATIONS AND DISCUSSION}

Nonallelic interactions and heterosis

The experimental design of the $8 \times 8$ diallel set of crosses between inbred varieties of $N$. rustica has been described in detail elsewhere. In general only the two seasons 1952 and 53, when parents, $F_{1}$ 's, $F_{2}$ 's, and backcrosses were grown simultaneously, concern us here.

For the data collected in both seasons the analysis of the family means for the character final height showed apparent overdominance and deviation of the $W_{r} / V_{r}$ regression from a linear slope of 1 , hence there was disagreement with the assumption of independence of the genes (JiNks 1954, 1956). The suggestion that this failure of the assumption was due to the dependence of the genes in their action (JrNKs 1954) i.e. nonallelic interaction, was borne out by the scaling tests of MATHER (1949) and an analysis of the components of variation (Jinks 1956).

The A, B and C scaling tests of MATHER were combined into a joint test using the squared standard deviations of the generation means averaged over all crosses as weights (CAVALLI 1952). This joint test detected 10 crosses showing significant ( $P$ « 0.05) nonallelic interaction in 1952 and the same 10 plus 2 others in 1953 (Jinks 1956). However, while this test allowed for different nonheritable components of variation in different generations, it did not allow for differences between crosses for the magnitude of this component. Reanalyses have therefore been made for comparison, using the variance of the generation means appropriate to each individual cross. The degrees of freedom available for estimating the nonheritable components of each generation mean for a single cross in our experimental design are as follows:-

Degrees of freedom of the nonheritable components of variation

\begin{tabular}{|c|c|c|c|c|c|}
\hline $\begin{array}{l}\text { Generation } \\
\text { mean }\end{array}$ & $\begin{array}{l}\text { Reciprocal } \\
\text { differences }\end{array}$ & $\begin{array}{l}\text { Duplicate } \\
\text { plots }\end{array}$ & Blocks & $\begin{array}{l}\text { Recips. } \\
\times \text { blocks }\end{array}$ & $\begin{array}{l}\text { Total } \\
\text { degrees of } \\
\text { freedom }\end{array}$ \\
\hline$\overline{P_{1}}$ and $\overline{P_{2}}$ & . & $2^{*}$ & 1 & . & 3 \\
\hline$\overline{\mathrm{F}_{1}} \ldots$ & 1 & . & 1 & 1 & 3 \\
\hline$\overline{\mathrm{F}_{2}^{\prime}} \ldots$ & 1 & 4 & 1 & 1 & 7 \\
\hline $\overrightarrow{\mathrm{B}}_{1}$ and $\overline{\mathrm{B}}_{2}$ & 1 & . & 1 & 1 & 3 \\
\hline
\end{tabular}

* Only $1 d f$ available in 1952 making total for each parent $\overline{P_{1}}$ and $\overline{P_{z}} 2 d f$.

Since our comparisons will be between the means of plots, the degrees of freedom for the nonheritable components of variation are based on comparisons between replicate plot means of each generation grown in each season. Every plot, however, contains five plants, so that in all we have 20 plants of each parent (ten 
in 1952), $F_{1}$ and backcross $\left(B_{1}\right.$ and $\left.B_{2}\right)$ generation and $40 F_{2}$ plants for each cross in each season.

The estimated variances and their degrees of freedom for the scaling tests are given by Mather (1949). For example, the variance for the $C$ scaling test is given by $V_{C}=V_{\overline{P_{1}}}+V_{\overline{P_{2}}}+4 V_{\overline{F_{1}}}+16 V_{\overline{F_{2}}}$ in our own case for 12 and 16 degrees of freedom in 1952 and 1953 respectively.

The same type of expectation holds for the components of the generation means. Thus the variance of $[i]$ is given by $V_{[i]}=4 V_{B_{1}}+4 V_{B_{2}}+16 V_{\overline{F_{2}}}$ for 13 degrees of freedom in both seasons.

Before, however, proceeding with the scaling tests and the estimation of the components of the generation means the sums of squares corresponding with the degrees of freedom listed above were estimated for each of the 28 crosses. These were pooled to give the variance of each of the generation means only if they were homogeneous.

A comparison of the crosses which show significant nonallelic interaction $(P$ < 0.05$)$ in these new tests and those which were significant in the earlier joint scaling tests in 1952 is given in Table 1.

All the ten crosses which showed nonallelic interactions on the joint scaling test show high significance for at least one of the individual scaling tests using errors appropriate to the generation means of individual crosses. In addition, four crosses which were not significant on the joint scaling test now show significance for one or other of the individual scaling tests.

Overall, therefore, 14 of the 28 crosses in 1952 give some indication of significant nonallelic interactions. For 1953 the overall total is 17 compared with 12 on

TABLE 1

The incidence of nonallelic interactions $(+)$ as detected by the scaling tests for 1952

\begin{tabular}{|c|c|c|c|c|}
\hline Cross & $\mathbf{A}$ & $\underset{B}{\text { Scaling tests }}$ & $\mathrm{C}$ & $\begin{array}{l}\text { Joint scaling test } \\
(\text { JINKS 1956) }\end{array}$ \\
\hline $1 \times 2 \ldots \ldots \ldots \ldots \ldots \ldots \ldots$ & + & + & + & + \\
\hline $1 \times 4, \ldots \ldots \ldots \ldots \ldots$ & - & - & + & + \\
\hline $1 \times 8, \ldots \ldots \ldots \ldots \ldots \ldots$ & - & - & + & + \\
\hline $2 \times 3 \ldots \ldots \ldots \ldots \ldots \ldots$ & + & + & - & + \\
\hline $2 \times 4 \ldots \ldots \ldots \ldots \ldots \ldots$ & - & + & + & - \\
\hline $2 \times 5 \ldots \ldots \ldots \ldots \ldots \ldots$ & + & - & - & - \\
\hline $2 \times 6 \ldots \ldots \ldots \ldots \ldots$ & + & - & - & + \\
\hline $2 \times 8 \ldots \ldots \ldots \ldots \ldots \ldots$ & - & + & + & + \\
\hline $3 \times 4, \ldots \ldots \ldots \ldots \ldots, \ldots$ & - & - & + & + \\
\hline $3 \times 8 \ldots \ldots \ldots \ldots \ldots \ldots$ & + & + & - & + \\
\hline $4 \times 6 \ldots \ldots \ldots \ldots \ldots$ & + & - & + & + \\
\hline $4 \times 8 \ldots \ldots \ldots \ldots \ldots \ldots$ & + & - & + & + \\
\hline $5 \times 6 \ldots \ldots \ldots \ldots \ldots \ldots$ & + & - & - & - \\
\hline $6 \times 8 \ldots \ldots \ldots \ldots \ldots \ldots$ & + & - & - & $-i$ \\
\hline Total $\ldots \ldots \ldots \ldots \ldots \ldots$ & 9 & 5 & 8 & 10 \\
\hline
\end{tabular}


the earlier joint scaling tests. Of the 14 crosses in 1952 and the 17 in 1953 showing some indication of nonallelic interaction, 13 crosses are common to both seasons.

We can now make a four way classification of the 28 crosses in each of the two seasons, into interacters and noninteracters, and into those showing significant or no significant heterosis (Table 2 ).

TABLE 2

The incidence of heterosis and nonallelic interaction

\begin{tabular}{|c|c|c|c|c|c|c|c|}
\hline \multirow{3}{*}{ Significant heterosis } & \multirow{3}{*}{$\begin{array}{l}\text { Season } \\
\ldots \ldots\end{array}$} & \multicolumn{3}{|c|}{1952 Non } & \multicolumn{2}{|c|}{1953} & \\
\hline & & Interacters & \multicolumn{2}{|c|}{ interacters } & \multirow{2}{*}{$\frac{\text { Interacters }}{11}$} & & \\
\hline & & 8 & 1 & 9 & & 4 & 15 \\
\hline No significant heterosis & $\ldots$ & 6 & 13 & 19 & 6 & 7 & 13 \\
\hline & & 14 & 14 & 28 & 17 & 11 & 28 \\
\hline
\end{tabular}

Taking both seasons together, there is a clear association between nonallelic interaction and heterosis, and the absence of nonallelic interaction and no heterosis $\left(x_{[1]}^{2}=9.6\right)$. This association is more marked in 1952 when the general level of heterosis was lower. Overall 60 percent of the interacting crosses show significant heterosis while only 20 percent of noninteracting crosses do so: or to put it another way, 80 percent of all heterotic crosses show significant nonallelic interactions.

This high correlation between the presence of nonallelic interaction and heterosis is even more marked when we consider the average heterosis of the crosses falling in each of these 4 classes. (Table 3)

TABLE 3

The incidence of nonallelic interactions and the average heterosis in inches

\begin{tabular}{|c|c|c|c|c|c|}
\hline & \multirow[t]{2}{*}{ Season } & \multicolumn{2}{|c|}{1952} & \multicolumn{2}{|c|}{1953} \\
\hline & & Interacters & $\begin{array}{c}\text { Non } \\
\text { interacters }\end{array}$ & Interacters & $\begin{array}{c}\text { Non } \\
\text { interacters }\end{array}$ \\
\hline Significant heterosis ... & $\ldots \ldots$ & $8 \cdot 81$ & $5 \cdot 60$ & $13 \cdot 68$ & 5.03 \\
\hline No significant heterosis & 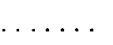 & 1.49 & 0.02 & 1.24 & 0.37 \\
\hline Mean ......... & & 5.40 & 0.61 & 9.02 & 2.32 \\
\hline
\end{tabular}

If we now consider the seasons jointly, then 70 percent of the crosses showing nonallelic interactions also show significant heterosis. In fact only one cross which consistently shows nonallelic interaction consistently shows no significant heterosis.

While stressing this correlation between the incidence of nonallelic interaction and heterosis, it is obvious that heterosis can arise, albeit at a lower frequency, and with a lower mean expression, in the absence of significant nonallelic inter- 
action, and further, that all crosses showing nonallelic interaction do not necessarily show heterosis.

\section{Estimates of the components of the generation means}

All six components of the generation means and their standard deviations have been estimated for all 28 crosses in both seasons. For those crosses where neither the components of nonallelic interaction nor any of the scaling tests are significantly different from zero, least squares estimates of the remaining components [d] $[h]$ and $M$ and their standard deviations have been obtained. Taking the latter crosses first, we find the following values for the additive [d] and dominance $[h]$ components of the generation means (Table 4 ).

TABLE 4,

Least squares estimates of the additive [d] and dominance $[\mathrm{h}]$ components of the generation means for those cases showing no nonallelic interactions

\begin{tabular}{|c|c|c|c|c|c|c|}
\hline Cross & Season $_{[d]}$ & $\begin{array}{l}1952 \\
{[h]}\end{array}$ & Heterosis & {$[d]$} & $\begin{array}{c}1953 \\
{[h]}\end{array}$ & Heterosis \\
\hline $1 \times 3$ & $2 \cdot 6 \pm 2 \cdot 0$ & $8.7 \pm 3.8$ & $5 \cdot 6 \pm 1 \cdot 4^{*}$ & $1.5 \pm 2 \cdot 6$ & $3.7 \pm 4.9$ & $1 \cdot 4 \pm 2 \cdot 2$ \\
\hline $1 \times 5$ & $1 \cdot 1 \pm 1 \cdot 8$ & $5.4 \pm 3.4$ & $4.4 \pm 1 \cdot 6$ & $2 \cdot 1 \pm 2 \cdot 0$ & $6.8 \pm 3.7$ & $4 \cdot 3 \pm 2 \cdot 0$ \\
\hline $1 \times 6$ & $2.7 \pm 1.9$ & $-0.6 \pm 3.6$ & $-3.4 \pm 1.4$ & $0.6 \pm 2.5$ & $1.6 \pm 4.6$ & $1.7 \pm 2 \cdot 1$ \\
\hline $1 \times 7$ & $0.7 \pm 1.7$ & $-0.4 \pm 3.2$ & $-1.4 \pm 0.9$ & \multicolumn{3}{|c|}{ Significant nonallelic interaction } \\
\hline $2 \times 7$ & $2 \cdot 1 \pm 3 \cdot 1$ & $4 \cdot 0 \pm 5.8$ & $2 \cdot 1 \pm 2 \cdot 6$ & $9 \cdot 1 \pm 2 \cdot 4$ & $2.7 \pm 4.5$ & $-5 \cdot 6 \pm 2 \cdot 0$ \\
\hline $3 \times 5$ & $4.1 \pm 1.9$ & $8 \cdot 1 \pm 3 \cdot 6$ & $3 \cdot 7 \pm 1 \cdot 5$ & \multicolumn{3}{|c|}{ Significant nonallelic interaction } \\
\hline $3 \times 6$ & $5 \cdot 5 \pm 2.3$ & $3 \cdot 1 \pm 4 \cdot 3$ & $-2.9 \pm 1.2$ & $1.8 \pm 1.6$ & $1.5 \pm 3.0$ & $-1 \cdot 3 \pm 1 \cdot 3$ \\
\hline $3 \times 7$ & $3.6 \pm 1.5$ & $3.6 \pm 2.9$ & $-0.5 \pm 1.1$ & $2 \cdot 3 \pm 2 \cdot 1$ & $-0.8 \pm 3.9$ & $-2 \cdot 8 \pm 2 \cdot 1$ \\
\hline $4 \times 5$ & $7 \cdot 3 \pm 2 \cdot 6$ & $9.8 \pm 4.9$ & $1.9 \pm 1.6$ & $9 \cdot 5 \pm 3 \cdot 6$ & $17 \cdot 8 \pm 6 \cdot 7$ & $8 \cdot 3 \pm 1 \cdot 7^{*}$ \\
\hline $4 \times 7$ & $8 \cdot 0 \pm 1 \cdot 7$ & $6.4 \pm 3.1$ & $-0.7 \pm 1.6$ & \multicolumn{3}{|c|}{ Significant nonallelic interaction } \\
\hline $5 \times 6$ & \multicolumn{3}{|c|}{ Significant nonallelic interaction } & $2 \cdot 4 \pm 1 \cdot 9$ & $6.7 \pm 3.6$ & $3 \cdot 8 \pm 1 \cdot 5$ \\
\hline $5 \times 7$ & $1 \cdot 1 \pm 1.2$ & $0.8 \pm 3.7$ & $0.5 \pm 1.3$ & $2 \cdot 6 \pm 1 \cdot 9$ & $7 \cdot 0 \pm 3 \cdot 7$ & $4.5 \pm 1.9$ \\
\hline $5 \times 8$ & $1.9 \pm 1.7$ & $3 \cdot 0 \pm 3 \cdot 3$ & $1.9 \pm 1.7$ & \multicolumn{3}{|c|}{ Significant nonallelic interaction } \\
\hline $6 \times 7$ & $2 \cdot 2 \pm 1 \cdot 7$ & $-2 \cdot 9 \pm 3 \cdot 3$ & $-4 \cdot 2 \pm 1.4$ & $4 \cdot 9 \pm 1 \cdot 8$ & $8 \cdot 0 \pm 3 \cdot 3$ & $3 \cdot 6 \pm 1 \cdot 1^{*}$ \\
\hline $6 \times 8$ & \multicolumn{3}{|c|}{ Significant nonallelic interaction } & $2.3 \pm 1.5$ & $5 \cdot 1 \pm 2 \cdot 9$ & $3 \cdot 6 \pm 0.7^{*}$ \\
\hline $7 \times 8$ & $0.9 \pm 1.5$ & $-1.4 \pm 2.8$ & $-2.2 \pm 1.7$ & \multicolumn{3}{|c|}{ Significant nonallelic interaction } \\
\hline
\end{tabular}

* Significant heterosis at $P \ll 0 \cdot 05$.

None of the crosses in this table, i.e. the crosses which show no significant nonallelic interaction, show significant heterosis in both seasons. Three crosses $1 \times 3$, $4 \times 5$, and $6 \times 7$, however, do show significant heterosis in one or other of the two seasons without any suggestion of nonallelic interaction in either season. We must, therefore, consider the possibility that the significant heterosis in these crosses is the result of overdominance or the dispersion of dominant increasing alleles in the parental lines. In these three crosses the dominance component of the generation means $[h]$ varies from 1.6 to 3.3 times the value of the additive component $[d]$. It is clear that this situation could arise as a result of overdominance $(\Sigma h>\Sigma d)$, incomplete association of dominant increasing alleles in the parental lines $\left(r_{d}< \pm 1\right)$, or any combination of these two. Should, however, over- 
dominance be responsible for the heterosis, then the dominance $(H)$ component of variation should be greater than the additive $(D)$. Estimates of these two components of variation for the three crosses $1 \times 3,4 \times 5$ and $6 \times 7$ using the $\mathrm{F}_{2}$ and backcross variances (see MATHER 1949) give no indication of overdominance. An explanation based on dispersion of the dominant increasing alleles would, therefore, appear to be more in line with the observations.

The remaining consistently noninteracting crosses listed in Table 4 show no significant heterosis and hence require no further comment.

The interacting crosses, as we have seen, fall into two groups, according to whether or not they show significant heterosis. Examples of interacting crosses which show no significant heterosis are provided by $1 \times 7,1 \times 8,3 \times 8,5 \times 6$, $5 \times 8$, and examples of the estimates of the components of their generation means are listed in Table 5. The standard errors of these components are obtained from those of the generation means from which the components are estimated.

TABLE 5

Estimates of the components of the generation means and of heterosis for a number of crosses which show significant nonallelic interaction but no heterosis

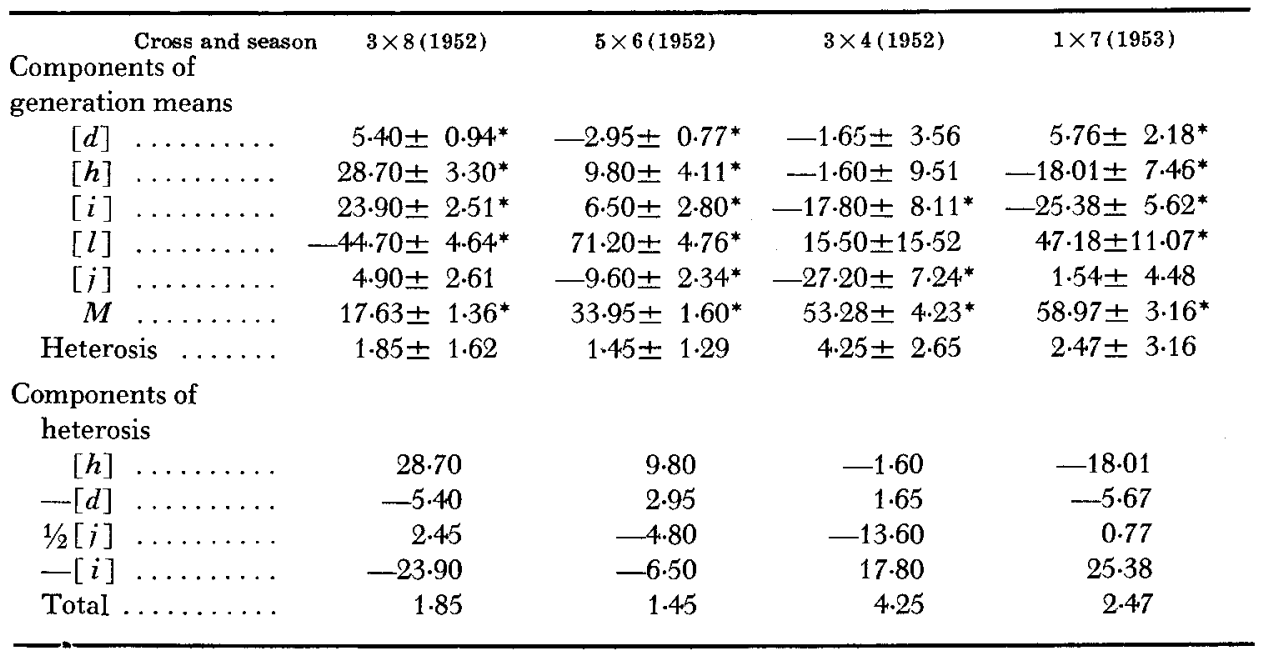

* Significant at $\mathrm{P} \ll 0.05$.

The four examples in Table 5 are typical of the situations that have been found in nonheterotic interacting crosses. In each of these crosses internal cancellation of the contributions of the four components of heterosis leads to an overall nonsignificant heterosis, although the individual components are in general highly significant.

In three of these crosses $[l]$ and $[h]$ have opposite signs suggesting duplicate type interactions. In cross $3 \times 4$, however, neither [l] nor [h] are significantly different from zero. Apart from the small contributions of $[d]$ and $1 / 2[j]$ it is the opposition of $[i]$ and $[h]$ which leads to the absence of heterosis in crosses $3 \times 8$, 
$5 \times 6$ and $1 \times 7$. In crosses $3 \times 8$ and $5 \times 6$, the positive contribution of $[h]$ is balanced by the negative one of [i] while in cross $1 \times 7$ it is the reverse. Furthermore the nonsignificant [j] component in crosses $3 \times 8$ and $1 \times 7$ suggest that either $r_{j}, \Sigma_{j}$ or both are very small or zero. If these interactions are in fact comparable with the duplicate types of classical genetics then it is $r_{j}$ which is small or zero, i.e. there is dispersion of the interacting genes in the parental lines. This would square with the sign of [i] relative to [h] which suggests that $q_{i}$ is negative. Overall, therefore, these interactions are compatible with dispersed duplicates.

Contrasting with this situation we have cross $3 \times 4$ where the absence of heterosis results from the opposition of the contributions of $[i]$ and $1 / 2[j]$. Clearly $r_{j}$ cannot be very small or zero, i.e. there is association of the interacting genes in the parental lines. The insignificance of $[h]$ and $[l]$ make them doubtful indicators of the type of interactions involved. Since neither classical complementary nor duplicate genes in association give heterosis in the $\mathrm{F}_{1}$, comparison with classical models provide no further clues as to the type of interaction involved in this cross.

Thus in the crosses which show nonallelic interactions but no heterosis, we find either opposition between the dominance and interaction components of heterosis, i.e. duplicate genes or association of the interacting genes in the parental lines.

Cross $1 \times 4$ may be taken as typical of many which show both heterosis and nonallelic interaction. It has been grown in every season from 1951 to 1956 (We are indebted to Dr. E. L. Breese for the observations in 1955 and 1956). From 1952 to $1956 \mathrm{~F}_{2}$ 's were included along with the parents and $F_{1}$ generations, while in 1952 and 1953 backcrosses were included and in 1956 one of the backcrosses $(1 \times 4) \times 1$ and $(4 \times 1) \times 1$ was grown. All the components of the generation means cannot be estimated in all the seasons, but estimates of certain combinations can be obtained from most of them (Table 6 ).

All estimates in Table 6 apart from the first and second in 1953 are significant at $\mathrm{P}$ « 0.05 . This low significance in 1953 can be traced to the large reciprocal cross and block differences, but apart from this there is a general consistency in sign and magnitude of the compound components of the generation means and heterosis over all seasons. Clearly the interpretation arrived at from a complete

TABLE 6

The components of the generation means for cross $1 \times 4$

\begin{tabular}{|c|c|c|c|c|c|c|c|c|}
\hline & Season & 1951 & 1952 & 1953 & 1954 & 1955 & 1956 & Mean \\
\hline Components & $2[i]+[l]$ & & -17.08 & -0.66 & -11.98 & -10.66 & -32.52 & \\
\hline \multirow{3}{*}{$\begin{array}{l}\text { Components } \\
\text { of } \\
\text { heterosis }\end{array}$} & {$[i]+[i]$} & $\ldots$ & -29.88 & -2.76 & & .. & -24.4 .1 & \\
\hline & {$[h]-[i]$} & 21.40 & 20.45 & 29.01 & 20.04 & 18.46 & $17 \cdot 18$ & 21.09 \\
\hline & $-[d]+1 / 2[j]$ & -7.20 & -8.90 & -7.86 & $-3 \cdot 31$ & -10.75 & -4.86 & $-7 \cdot 15$ \\
\hline Heterosis & & 14.20 & 11.55 & 21.15 & 16.73 & 7.72 & 12.32 & 13.94 \\
\hline
\end{tabular}


analysis in 1952 will not differ in detail from that which would apply in the other seasons.

In 1952 the estimated components of heterosis are as follows:

Component $\quad[h]-[i]-[d]+1 / 2[j]=$ Heterosis

Estimate $4.25+16.20-2.05-6.85=11.55$

Compound

$$
\underbrace{25+10}_{20 \cdot 45}-\underbrace{05}_{8 \cdot 90}
$$

estimate from

Table 6.

Clearly heterosis in this cross results from reinforcing dominance and nonallelic interactions; the latter making the major contribution, and apart from 1953 where the nonsignificance of many components makes interpretation difficult, this situation appears to hold in the other seasons.

The closest comparable classical interaction to that found in cross $1 \times 4$ is, of course, a complementary type with the interacting genes preponderantly dispersed in the parental lines.

Most interacting crosses showing heterosis are of this type, but contrasting with them is a situation which is at its most extreme in cross $1 \times 2$. The components of the generation means of the latter are listed in Table 7.

For 1953 the two blocks are given separately as the generation means differed significantly more between blocks than did duplicate or reciprocal plots within a block.

In 1952 there is apparently complete dispersion of duplicate genes, the heterosis resulting from the excess of the positive dominance $[h]$ over the negative interaction [i] contribution. In block I of 1953 the situation is comparable with cross $1 \times 4$ (Table 6) i.e. a complementary type interaction with the interacting genes dispersed in the parental lines, the heterosis resulting from the interaction component $[i]$. Block II in the same season, however, is intermediate; the significant $[j]$ component suggesting association. In the absence of a significant $[l]$ either a duplicate or complementary type of interaction would equally well fit the data.

TABLE 7

The components of the generation means for the cross $1 \times 2$

\begin{tabular}{|c|c|c|c|}
\hline & Season & $1953 \mathrm{I}$ & 1953 II \\
\hline \multicolumn{4}{|l|}{ Components } \\
\hline$[d] \ldots \ldots \ldots \ldots$ & $0.00 \pm 2.45$ & $2 \cdot 00 \pm 2 \cdot 69$ & $-2.70 \pm 0.61^{*}$ \\
\hline$[h] \ldots \ldots \ldots \ldots \ldots$ & $53 \cdot 19 \pm 9.89^{*}$ & $4.58 \pm 13.15$ & $22.05 \pm 6.45^{*}$ \\
\hline$[i] \ldots \ldots \ldots \ldots \ldots$ & $38.10 \pm 7.43^{*}$ & $-19.40 \pm 8 \cdot 78^{*}$ & $2 \cdot 40 \pm 5 \cdot 17$ \\
\hline$[l] \ldots \ldots \ldots \ldots \ldots$ & $-60 \cdot 72 \pm 11.89^{*}$ & $51.45 \pm 14.12^{*}$ & $-11.10 \pm 8.53$ \\
\hline$[j] \ldots \ldots \ldots \ldots \ldots$ & $-4.80 \pm 5.16$ & $-1.95 \pm 5.94$ & $-14.70 \pm 3.42^{*}$ \\
\hline$M \ldots \ldots \ldots$ & $12 \cdot 98 \pm 3.83^{*}$ & $60 \cdot 68 \pm 6 \cdot 04^{*}$ & $54.77 \pm 4.15^{*}$ \\
\hline Heterosis . . . . . . . & $12 \cdot 69 \pm 1.97^{*}$ & $21.00 \pm 3.81^{*}$ & $15 \cdot 00 \pm 3.01^{*}$ \\
\hline
\end{tabular}


Clearly the genotype environmental interaction in this cross is such that a consistent interpretation of the basis of the heterosis is impossible. We can, however, profitably examine the nature of this interaction.

Between the three environments the magnitude of the heterosis in cross $1 \times 2$ is positively correlated with the contribution of $[i]$, and negatively correlated with the contribution of $[h]$ to the heterosis. Superficially, therefore, in those environments which allow a fuller expression of the heterotic potential of this cross, nonallelic interactions play the major role in its production. We can now enquire as to the nature of an environmental restriction to the development of heterosis which would lead to a change from a complementary to a duplicate type of nonallelic interaction.

An environmental restriction to the development of the full height potential of the parents and offspring of cross $1 \times 2$ would effect the $F_{1}$ more than any other generation. Similarly, the backcross to the larger parent would be affected more than that to the smaller parent. If we impose such restrictions on a classical complementary system, sooner or later, we obtain estimates of the generation means which resemble those obtained from a duplicate or intermediate type of interaction. Furthermore, changes of scale e.g. antilogs, lead to the same type of change. Environmental restrictions on the attainment of the full height potential, therefore, provide an adequate explanation of the apparent inconsistency of the genetical basis of heterosis in cross $1 \times 2$.

\section{CONCLUSIONS}

Heterosis is a complex genetical phenomenon depending on the balance of the additive, dominance and interaction between homozygous/homozygotes and homozygous/heterozygous components of the generation means as well as on the distribution of the genes in the parental lines. Strangely enough it does not depend on the interaction between heterozygous/heterozygous combinations, but not so strange when we remember that heterosis is but the reverse counterpart of inbreeding depression.

As a consequence of its complexity the presence or absence of heterosis is not in itself indicative of the presence or absence of any particular type of gene action or interaction; it can result from a whole range of combinations of gene effects, many of which have been illustrated by our $N$. rustica data. Nevertheless, there is a correlation between the presence or absence of heterosis and the presence or absence of nonallelic interactions, and while heterosis can arise in the absence of nonallelic interactions it does so with a lower frequency and a lower mean expression.

\section{SUMMARY}

Heterosis can be expressed in terms of four of the components of the generation means of a cross between two inbred lines. These components can be estimated from the means of the parents, $F_{1}$ 's and $F_{2}$ 's and backcross generations of such a cross. This allows an assessment of the relative roles of dominance, nonallelic interaction and gene distribution in the production of heterosis and also a 
preliminary classification of the interaction into a complementary or duplicate type.

The method is illustrated by an analysis of the components of heterosis for final height in 28 crosses between inbred varieties of $N$. rustica.

\section{LITERATURE CITED}

Allard, R. W., 1956 Estimation of prepotency from Lima Bean diallel cross data. Agron. J. 48: $537-543$.

Cavalli, L. L., 1952 An analysis of linkage in quantitative inheritance. p. 135-144. Quantitative Inheritance. H.M.S.O. London.

Dickrnson, A. G., and J. L. Jinks, 1956 A generalised analysis of diallel crosses. Genetics 41: 65-78.

Hayman, B. I., 1954 The theory and analysis of diallel crosses. Genetics 39: 789-809.

Hayman, B. I., and K. Matrer, 1955 The description of genetic interactions in continuous variation. Biometrics 11: 69-82.

JiNks, J. L., 1954 The analysis of continuous variation in a diallel cross of Nicotiana rustica varieties. Genetics 39 : 767-788.

1955 A survey of the genetical basis of heterosis in a variety of diallel crosses. Heredity 9: 223-238.

1956 The $\mathrm{F}_{2}$ and backcross generation from a set of diallel crosses. Heredity 10: 1-30.

Jinks, J. L., and B. I. HaYman, 1953 Analysis of diallel crosses. Maize Genetics Co-operation News Letter 27 : 48-54.

MATHER, K., 1949 Biometrical Genetics. Methuen. London. 